\title{
OPEN A retrospective study on the therapeutic effects of sodium bicarbonate for adult in-hospital cardiac arrest
}

\author{
Chih-Hung Wang ${ }^{1,2}$, Cheng-Yi Wu ${ }^{1}$, Meng-Che Wu ${ }^{1}$, Wei-Tien Chang ${ }^{1,2}$, Chien-Hua Huang ${ }^{1,2}$, \\ Min-Shan Tsai ${ }^{1,2}$, Tsung-Chien Lu ${ }^{1,2}$, Eric Chou ${ }^{3}$, Yu-Lin Hsieh ${ }^{4}$ \& Wen-Jone Chen ${ }^{1,2,5 凶}$
}

To investigate whether the effects of sodium bicarbonate (SB) during cardiopulmonary resuscitation (CPR) would be influenced by blood $\mathrm{pH}$ and administration timing. Adult patients experiencing in-hospital cardiac arrest (IHCA) from 2006 to 2015 were retrospectively screened. Early intra-arrest blood gas data were obtained within $10 \mathrm{~min}$ of CPR. Multivariable logistic regression analysis and generalised additive models were used for effect estimation and data exploration, respectively. A total of 1060 patients were included. Only 59 patients demonstrated favourable neurological status at hospital discharge. Blood $\mathrm{pH} \leq 7.18$ was inversely associated with favourable neurological outcome (odds ratio [OR], 0.24; 95\% confidence interval [CI], $0.11-0.52 ; p$ value $<0.001$ ) while $\mathrm{SB}$ use was not. In the interaction analysis for favourable neurological outcome, significant interactions were noted between SB use and time to SB (SB use $\times$ time to SB $\geq 20 \mathrm{~min}$; OR 6.16; 95\% CI 1.42-26.75; $p$ value $\mathbf{0 . 0 2}$ ). In the interaction analysis for survival to hospital discharge, significant interactions were noted between SB use and blood pH (Non-SB use $\times$ blood pH > 7.18; OR 1.56; 95\% CI 1.01-2.41; $p$ value $\mathbf{0 . 0 5}$ ). SB should not be empirically administered for patients with IHCA since its effects may be influenced by blood $\mathrm{pH}$ and administration timing.

Annually, a total of 209,000 patients sustain in-hospital cardiac arrest (IHCA) in the United States ${ }^{1}$. Approximately $24 \%$ of patients with IHCA survive to hospital discharge; among these patients, $14 \%$ demonstrate severe neurological disability ${ }^{1}$.

Cardiac arrest may lead to severe acidosis ${ }^{2-4}$ because of increased anaerobic metabolism and cessation of pulmonary gas exchange. Buffering acidosis with sodium bicarbonate (SB) was intuitive; therefore, it had been recommended as a first-line treatment for cardiopulmonary resuscitation $(\mathrm{CPR})^{5}$. Nevertheless, SB administration during CPR was not without risks, such as worsening intracellular acidosis ${ }^{6}$, which may cause worse outcomes.

The guidelines ${ }^{6,7}$ did not recommend routine use of SB during CPR, except for hyperkalaemia-related arrest and tricyclic overdose. Using the American Heart Association Get With The Guidelines-Resuscitation database, Moskowitz et al. ${ }^{8}$ found a significant increase in SB use for IHCA between the years 2001 and 2016. Compared to the year 2001, patients with IHCA in the year 2016 had two-fold higher odds of receiving $\mathrm{SB}^{8}$, which resulted in half of them receiving SB during $\mathrm{CPR}^{8}$. This suggests $\mathrm{SB}$ may be used beyond the guideline-recommended indications $s^{6,7}$.

A small retrospective study ${ }^{9}$ revealed that approximately $31 \%$ (27 out of 88 ) of patients with IHCA received SB during CPR without any blood gas data and $11 \%$ (10 out of 88 ) of patients receiving the first SB injection empirically were later found to have alkalemia. Furthermore, a large retrospective study ${ }^{10}$ demonstrated that SB was often given late during CPR, probably as a "last-ditch effort," which could have biased the results toward harmful association ${ }^{11}$. Therefore, it was probably not SB that worsened CPR outcomes. It just failed to improve outcomes.

Early blood gas analysis may reduce the frequency of unwarranted use of SB and clarify its effects during CPR. Therefore, in this exploratory analysis, we attempted to (1) investigate the therapeutic effects of SB during

\footnotetext{
${ }^{1}$ Department of Emergency Medicine, Zhongzheng Dist, National Taiwan University Hospital, No.7, Zhongshan S. Rd, Taipei City 100, Taiwan, ROC. ${ }^{2}$ Department of Emergency Medicine, College of Medicine, National Taiwan University, Taipei City, Taiwan, ROC. ${ }^{3}$ Department of Emergency Medicine, Baylor Scott and White All Saints Medical Center, Fort Worth, TX, USA. ${ }^{2}$ Department of Internal Medicine, Danbury Hospital, Danbury, CT, USA. ${ }^{5}$ Division of Cardiology, Department of Internal Medicine, National Taiwan University Hospital and National Taiwan University College of Medicine, Taipei City, Taiwan, ROC. ${ }^{\circledR}$ email: wjchen1955@ntu.edu.tw
} 
CPR, and (2) explored whether the effects of SB would be influenced by intra-arrest blood pH and timing of administration in the interaction analysis, thereby identifying the optimal indications for SB use during CPR.

\section{Materials and methods}

Setting. We used previously established IHCA database for analysis ${ }^{12,13}$. Briefly, this retrospective study was conducted at National Taiwan University Hospital (NTUH), a tertiary medical centre, which had 2600 beds, including 220 beds in intensive care units (ICUs). This study was performed in accordance with the Declaration of Helsinki amendments and approved by the Research Ethics Committee of the NTUH (reference number: 201805098RINC). The requirement for informed consent was waived because of the retrospective and noninterventional study design. In NTUH, a code team is activated whenever a cardiac arrest event occurs in the general wards. For IHCA in the ICUs, resuscitation is performed by the ICU staff without activating the code team. CPR is performed according to guideline recommendations ${ }^{14,15}$. In NTUH, point-of-care blood gas analysers are deployed in every ward floor, including floor of general wards and ICUs. Physicians are instructed to obtain blood samples as soon as possible for blood gas analysis in order to identify potential causes leading to IHCA.

Participants. Patients sustaining IHCA at NTUH between 2006 and 2015 were screened by the following inclusion criteria: (1) age $\geq 18$ years; (2) receiving CPR for $\geq 2 \mathrm{~min}$; (3) absence of a do-not-resuscitate order before arrest and (4) early intra-arrest blood gas analysis with available blood $\mathrm{pH}$, partial pressure of carbon dioxide $\left(\mathrm{PCO}_{2}\right)$ and bicarbonate $\left(\mathrm{HCO}_{3}{ }^{-}\right)$data. If a single patient suffered from multiple IHCA events during hospitalisation, only the first event was analysed. Trauma-related IHCA was excluded. Patients were also excluded if the timing of SB use was not documented.

Data collection and outcome measures. The following information was abstracted for each patient: age, sex, comorbidities, variables suggested by the Utstein template ${ }^{16}$, timing of SB use, early intra-arrest blood gas analysis data and critical interventions performed at the time of cardiac arrest and after sustained return of spontaneous circulation (ROSC). Sustained ROSC was defined as ROSC lasting for 20 min consecutively without resuming repeated CPR. Early intra-arrest blood gas analysis, including measurement of blood $\mathrm{pH}, \mathrm{PCO}_{2}$ and $\mathrm{HCO}_{3}^{-}$, was defined as the first available blood gas data measured within $10 \mathrm{~min}$ of initiating $\mathrm{CPR}$, which was usually obtained in the beginning of CPR from the arterial source. Severe metabolic acidaemia was defined as blood $\mathrm{pH} \leq 7.2, \mathrm{PaCO}_{2} \leq 45 \mathrm{~mm} \mathrm{Hg}$ and $\mathrm{HCO}_{3}{ }^{-}$concentration $\leq 20 \mathrm{mmol} / \mathrm{L}^{17}$. Time to $\mathrm{SB}$ was defined as elapsed time from the first chest compression to the first time of SB administration. Duration of CPR was defined as the period from the initiation of chest compression to the termination of resuscitation attempts, either due to sustained ROSC or due to declaration of death.

The primary outcome was favourable neurological recovery at hospital discharge, defined as a Cerebral Performance Category scale score of 1 or $2^{18}$. The secondary outcome was survival to hospital discharge. The Cerebral Performance Category scale score was determined by retrospectively reviewing the medical records of each patient.

Statistical analysis. Categorical variables are expressed as counts with percentages, while continuous variables are expressed as medians with interquartile ranges. Categorical and continuous variables were compared by Chi-squared test and Wilcoxon rank-sum test, respectively.

The odds ratio (OR) was designated as the outcome measure. Univariate and multivariable logistic regression analyses were conducted to investigate the associations between independent variables and outcomes. All available independent variables were accounted for in the regression model, regardless of whether they were considered as significant in univariate analyses. Generalised additive models (GAMs) ${ }^{19}$ were employed to explore nonlinear effects of the continuous variables on outcomes and to define the potential cut-off points for transforming a continuous variable into a categorical variable during the model-fitting process. The stepwise variable selection procedure with iterations between the forward and backward steps was utilized to build the final regression model. Significance levels for entry and to stay were defined at 0.15 to avoid exclusion of candidate variables. The final regression model was obtained by sequentially excluding individual variables with a $p$ value $>0.05$ until all regression coefficients were significant. If the variable of interest, SB use, was not included in the final model, this variable would be forcibly entered into the model to assess its effect estimate.

In the prespecified interaction analyses, the interactions of $\mathrm{SB}$ use with blood $\mathrm{pH}$, severe metabolic acidaemia and time to SB were assessed. The goodness-of-fit of the fitted regression model was examined using $c$ statistics, the adjusted generalised $R^{2}$ and the Hosmer-Lemeshow goodness-of-fit test. Analysis was performed using R 3.3.1 software (R Foundation for Statistical Computing, Vienna, Austria). A two-tailed $p$ value $<0.05$ was considered significant.

\section{Results}

Between 2006 and 2015, a total of 1698 adult non-trauma patients receiving CPR for $\geq 2$ min after IHCA were screened. Of these, 599 patients were excluded due to the lack of early intra-arrest blood gas analysis; 39 patients were further excluded because the timing of SB use was not documented. Finally, a total of 1060 patients were included for further analysis. The differences between patients included and excluded from the analysis were shown in Supplemental Tables 1 and 2.

The characteristics of the included patients are provided in Tables 1 and 2. Median patient age was 68.2 years. Median CPR duration was $30 \mathrm{~min}$. A total of 733 patients (69.2\%) received SB during CPR and the median time to $\mathrm{SB}$ was $3.7 \mathrm{~min}$ (The differences between patients with and without SB use were shown in Supplemental Tables 3 


\begin{tabular}{|c|c|c|c|c|c|}
\hline Variables & All Patients $(n=1060)$ & $\begin{array}{l}\text { Patients With Favourable } \\
\text { Neurological Outcome at } \\
\text { Hospital Discharge }(n=59)\end{array}$ & $\begin{array}{l}\text { Patients Without Favourable } \\
\text { Neurological Outcome at } \\
\text { Hospital Discharge }(n=1001)\end{array}$ & $p$ value & $\begin{array}{l}\text { Odds ratio ( } 95 \% \text { confidence } \\
\text { interval) }\end{array}$ \\
\hline Age, years, median (IQR) & $68.2(56.5-78.9)$ & $62.6(53.2-73.6)$ & $68.5(56.9-79.4)$ & 0.007 & $0.98(0.97-1.00)$ \\
\hline Male, n (\%) & $649(61.2)$ & $44(80.0)$ & $605(60.4)$ & 0.03 & $1.92(1.03-3.76)$ \\
\hline \multicolumn{6}{|l|}{ Comorbidities, n (\%) } \\
\hline Heart failure, this admission & $208(19.6)$ & $17(28.8)$ & $191(19.1)$ & 0.07 & $1.72(0.90-3.16)$ \\
\hline Heart failure, prior admission & $171(16.1)$ & $11(18.6)$ & $160(16.0)$ & 0.59 & $1.20(0.55-2.42)$ \\
\hline $\begin{array}{l}\text { Myocardial infarction, this } \\
\text { admission }\end{array}$ & $120(11.3)$ & $12(20.3)$ & $108(10.8)$ & 0.02 & $2.11(0.99-4.20)$ \\
\hline $\begin{array}{l}\text { Myocardial infarction, prior } \\
\text { admission }\end{array}$ & $39(3.7)$ & $4(6.8)$ & $35(3.5)$ & 0.19 & $2.01(0.50-5.91)$ \\
\hline Arrhythmia & $192(18.1)$ & $9(15.3)$ & $183(18.3)$ & 0.56 & $0.80(0.34-1.69)$ \\
\hline Hypotension & $260(24.5)$ & $12(20.3)$ & $248(24.8)$ & 0.44 & $0.78(0.37-1.51)$ \\
\hline Respiratory insufficiency & $764(72.1)$ & $37(62.7)$ & $727(72.6)$ & 0.10 & $0.63(0.36-1.15)$ \\
\hline Renal insufficiency & $446(42.1)$ & $24(40.7)$ & $422(42.2)$ & 0.82 & $0.94(0.53-1.65)$ \\
\hline Hepatic insufficiency & $182(17.2)$ & $8(13.6)$ & $174(17.4)$ & 0.45 & $0.75(0.30-1.62)$ \\
\hline $\begin{array}{l}\text { Metabolic or electrolyte abnor- } \\
\text { mality }\end{array}$ & $186(17.5)$ & $8(13.6)$ & $178(17.8)$ & 0.41 & $0.73(0.29-1.58)$ \\
\hline Diabetes mellitus & $354(33.4)$ & $17(28.8)$ & $337(33.7)$ & 0.44 & $0.80(0.42-1.46)$ \\
\hline $\begin{array}{l}\text { Baseline evidence of motor, cogni- } \\
\text { tive, or functional deficits }\end{array}$ & $329(31.0)$ & $13(22.0)$ & $316(31.6)$ & 0.12 & $0.61(0.30-1.17)$ \\
\hline Acute stroke & $45(4.2)$ & $1(1.7)$ & $44(4.4)$ & 0.32 & $0.38(0.01-2.29)$ \\
\hline $\begin{array}{l}\text { Favourable neurological status } \\
24 \text { h before cardiac arrest }\end{array}$ & $462(43.6)$ & $36(61.0)$ & $426(42.6)$ & 0.006 & $2.11(1.20-3.79)$ \\
\hline Pneumonia & $337(31.8)$ & $8(13.6)$ & $329(32.9)$ & 0.002 & $0.32(0.13-0.69)$ \\
\hline Bacteraemia & $86(8.1)$ & $0(0)$ & $86(8.6)$ & 0.02 & $0.09(0.006-1.45)$ \\
\hline Cirrhosis & $70(6.6)$ & $1(1.7)$ & $69(6.9)$ & 0.12 & $0.23(0.006-1.40)$ \\
\hline $\begin{array}{l}\text { Chronic obstructive pulmonary } \\
\text { disease }\end{array}$ & $62(5.8)$ & $4(6.8)$ & $58(5.8)$ & 0.75 & $1.18(0.30-3.37)$ \\
\hline Dialysis & $191(18.0)$ & $10(16.9)$ & $81(8.1)$ & 0.83 & $0.92(0.41-1.89)$ \\
\hline $\begin{array}{l}\text { Metastatic cancer or any blood- } \\
\text { borne malignancy }\end{array}$ & $247(23.3)$ & $4(6.8)$ & $243(24.3)$ & 0.002 & $0.23(0.06-0.62)$ \\
\hline $\begin{array}{l}\text { Charlson comorbidity index, } \\
\text { median (IQR) }\end{array}$ & $2(1-4)$ & $2(1-3.8)$ & $2(1-4)$ & 0.004 & $0.83(0.72-0.95)$ \\
\hline
\end{tabular}

Table 1. Baseline Characteristics of Study Patients. Abbreviations: IQR, interquartile range.

and 4). Median $\mathrm{pH}, \mathrm{PCO}_{2}$ and $\mathrm{HCO}_{3}{ }^{-}$levels were $7.2,50.1 \mathrm{~mm} \mathrm{Hg}$ and $20.2 \mathrm{mmol} / \mathrm{L}$, respectively. There were 115 patients (10.8\%) categorised as having severe metabolic acidaemia. Only 124 patients (11.7\%) survived to hospital discharge; of these, 59 patients (5.6\%) demonstrated favourable neurological status.

The GAM plots demonstrated the association of logit $(\mathrm{p})$, where $\mathrm{p}$ represented the probability for favourable neurological status, with blood pH (Fig. 1A) and time to SB administration (Fig. 1B). If logit (p) was ' 0 , the odds for favourable neurological status were > 1 . The cut-off points of blood $\mathrm{pH}$ value of 7.18 and time to SB of $20 \mathrm{~min}$ were used to transform blood $\mathrm{pH}$ and time to SB into binary variables during the model-fitting process.

For the primary outcome (Table 3 ), in the primary model, blood $\mathrm{pH} \leqq 7.18$ was inversely associated with favourable neurological outcome (OR $0.24 ; 95 \%$ confidence interval [CI] $0.11-0.52$; $p$ value $<0.001$ ) while SB use was not. In the interaction analysis, significant interactions were noted between SB use and blood $\mathrm{pH}$ (SB use $\times$ blood $\mathrm{pH} \leq 7.18$; OR $0.29 ; 95 \%$ CI $0.11-0.76$; $p$ value $=0.008$; non-SB use $\times$ blood $\mathrm{pH} \leq 7.18$; OR, 0.20; $95 \%$ CI $0.06-0.60 ; p$ value $=0.004$ ) or time to SB (SB use $\times$ time to $\mathrm{SB} \geq 20 \mathrm{~min}$; OR 6.16; 95\% CI 1.42-26.75; $p$ value $=0.02$ ), but no significant interaction was noted between $S B$ use and severe metabolic acidaemia.

For the secondary outcome (Table 4), in the secondary model, blood $\mathrm{pH} \leq 7.18$ was inversely associated with survival (OR 0.61; 95\% CI 0.38-0.98; $p$ value $=0.04)$ while SB use was not. In the interaction analysis, non-SB use $\times$ blood $\mathrm{pH}>7.18$ was positively associated with survival $(\mathrm{OR} 1.82 ; 95 \%$ CI $1.12-2.95$; $p$ value $=0.02$ ).

\section{Discussion}

Main findings. Overall, SB use was not associated with IHCA outcomes when the confounding effects of blood $\mathrm{pH}$ were adjusted. Blood $\mathrm{pH} \leq 7.18$ was significantly associated with worse outcomes. Therefore, by interaction analysis, we tested the hypothesis, i.e. whether the effect of SB would be different in different populations. These populations included patients with blood $\mathrm{pH} \leq 7.18$ versus $>7.18$, patients with versus without severe metabolic acidaemia and patients with CPR duration $<$ versus $\geq 20$ min. The results demonstrated that for patients with blood $\mathrm{pH}>7.18$, non-SB use was associated with survival while for patients with CPR duration $\geq 20 \mathrm{~min}$, $\mathrm{SB}$ use was associated with better neurological recovery. 


\begin{tabular}{|c|c|c|c|c|c|}
\hline Variables & All Patients $(n=1060)$ & $\begin{array}{l}\text { Patients With Favourable } \\
\text { Neurological Outcome at } \\
\text { Hospital Discharge }(n=59)\end{array}$ & $\begin{array}{l}\text { Patients Without Favourable } \\
\text { Neurological Outcome at } \\
\text { Hospital Discharge }(n=1001)\end{array}$ & $p$ value & $\begin{array}{l}\text { Odds ratio ( } 95 \% \text { confidence } \\
\text { interval) }\end{array}$ \\
\hline Arrest at night, $\mathrm{n}(\%)$ & $385(36.3)$ & $22(37.3)$ & $363(36.3)$ & 0.87 & $1.04(0.58-1.85)$ \\
\hline Arrest on weekend, n (\%) & $301(28.4)$ & $11(18.6)$ & $290(29.0)$ & 0.09 & $0.56(0.26-1.12)$ \\
\hline Arrest location, $\mathrm{n}(\%)$ & & & & 0.06 & $1.05(0.67-1.62)$ \\
\hline Intensive care unit & $473(44.6)$ & $29(49.2)$ & $444(44.4)$ & & \\
\hline General ward & $525(49.5)$ & $23(39.0)$ & $502(50.1)$ & & \\
\hline Others & $62(5.8)$ & $7(11.9)$ & $55(5.5)$ & & \\
\hline Witnessed arrest, n (\%) & $734(69.2)$ & $43(72.9)$ & $691(60.0)$ & 0.53 & $1.21(0.65-2.33)$ \\
\hline Monitored status, n (\%) & $645(60.8)$ & $42(71.2)$ & $603(60.2)$ & 0.09 & $1.63(0.89-3.10)$ \\
\hline Shockable rhythm, n (\%) & $147(13.9)$ & $20(33.9)$ & $127(12.7)$ & $<0.001$ & $3.52(1.88-6.42)$ \\
\hline \multicolumn{6}{|c|}{ Critical care interventions in place at time of arrest, $\mathrm{n}(\%)$} \\
\hline \begin{tabular}{l|l} 
Mechanical ventilation & \\
\end{tabular} & $267(25.2)$ & $14(23.7)$ & $253(25.3)$ & 0.79 & $0.92(0.46-1.74)$ \\
\hline Antiarrhythmics & $119(11.2)$ & $5(8.5)$ & $114(11.4)$ & 0.49 & $0.72(0.22-1.84)$ \\
\hline Vasopressors & $474(44.7)$ & $21(35.6)$ & $453(45.3)$ & 0.15 & $0.67(0.37-1.19)$ \\
\hline Dialysis & $78(7.4)$ & $3(5.1)$ & $75(7.5)$ & 0.49 & $0.66(0.13-2.11)$ \\
\hline Pulmonary artery catheter & $6(0.6)$ & $2(3.4)$ & $4(0.4)$ & 0.003 & $8.70(0.77-62.20)$ \\
\hline Intra-aortic balloon pumping & $8(0.8)$ & $1(1.7)$ & $7(0.7)$ & 0.39 & $2.45(0.05-19.55)$ \\
\hline CPR duration, min, median (IQR) & $30(14-49)$ & $9(5.3-18.8)$ & $31(15-50.3)$ & $<0.001$ & $0.92(0.90-0.95)$ \\
\hline \begin{tabular}{l|l} 
SB use, $\mathrm{n}(\%)$ \\
\end{tabular} & $733(69.2)$ & $27(48.5)$ & $706(70.5)$ & $<0.001$ & $0.35(0.20-0.62)$ \\
\hline Time to SB, median (IQR) & $3.7(0-6)(\mathrm{n}=733)$ & $3.4(0-4.5)(\mathrm{n}=27)$ & $3.8(0-6)(\mathrm{n}=706)$ & 0.45 & $1.00(0.96-1.05)$ \\
\hline \multicolumn{6}{|l|}{ Intra-arrest blood gas analysis } \\
\hline Blood pH, median (IQR) & $7.2(7.1-7.3)$ & $7.3(7.2-7.4)$ & $7.2(7.1-7.3)$ & $<0.001$ & $14.30(3.67-55.65)$ \\
\hline $\mathrm{PCO}_{2}, \mathrm{mmHg}$, median (IQR) & $50.1(35.0-74.0)$ & $41.1(32.3-57.0)$ & $51.3(35.0-75.0)$ & 0.004 & $0.99(0.97-1.00)$ \\
\hline $\mathrm{HCO}_{3}{ }^{-}, \mathrm{mmol} / \mathrm{L}$, median (IQR) & $20.2(14.6-25.5)$ & $20.7(16.9-24.7)$ & $20.1(14.6-25.5)$ & 0.43 & $1.00(0.98-1.02)$ \\
\hline Severe metabolic acidaemia, $\mathrm{n}(\%)$ & $115(10.8)$ & $5(8.5)$ & $110(11.0)$ & 0.55 & $0.75(0.29-1.92)$ \\
\hline \multicolumn{6}{|c|}{ Post-ROSC $\mathrm{e}^{\mathrm{e}}$ interventions, $\mathrm{n}(\%)$} \\
\hline $\begin{array}{l}\text { Extracorporeal membrane } \\
\text { oxygenation }\end{array}$ & $84(7.9)$ & $7(11.9)$ & $77(7.7)$ & 0.25 & $1.61(0.60-3.73)$ \\
\hline $\begin{array}{l}\text { Targeted temperature manage- } \\
\text { ment }\end{array}$ & $12(1.1)$ & $3(5.1)$ & $9(0.9)$ & 0.003 & $5.88(1.00-24.44)$ \\
\hline $\begin{array}{l}\text { Percutaneous coronary interven- } \\
\text { tion }\end{array}$ & $35(3.3)$ & $12(20.3)$ & $23(2.3)$ & $<0.001$ & $10.80(4.60-24.29)$ \\
\hline Sustained ROSC, n (\%) & $584(55.1)$ & $59(100)$ & $525(52.4)$ & $<0.001$ & $107.90(6.65-1750.19)$ \\
\hline $\begin{array}{l}\text { Survival to hospital discharge, } \\
\mathrm{n}(\%)\end{array}$ & $124(11.7)$ & $59(100)$ & $65(6.5)$ & $<0.001$ & $1701.43(104.01-27,832.32)$ \\
\hline
\end{tabular}

Table 2. Features, Interventions and Outcomes of Cardiac Arrest Events. Abbreviations: CPR, cardiopulmonary resuscitation; IQR, interquartile range; $\mathrm{SB}$, sodium bicarbonate; $\mathrm{PCO}_{2}$, partial pressure of carbon dioxide; ROSC, return of spontaneous circulation.

Comparisons with previous studies. Few studies investigated the effects of SB use during CPR for $\mathrm{IHCA}^{20}$. During CPR, point-of-care blood testing may yield important diagnostic information and guide management ${ }^{21}$. Compared with out-of-hospital cardiac arrest (OHCA), it may be easier for clinicians to diagnose acidosis for IHCA. In our study, about 65\% (1099 out of 1698) of screened patients had early intra-arrest blood gas analysis, revealing that $10.8 \%$ of them were experiencing severe metabolic acidaemia. Nonetheless, not only severe metabolic acidaemia, blood $\mathrm{pH} \leq 7.18$ itself was identified to be a significant predictor for IHCA outcomes. That is, severe acidosis (blood $\mathrm{pH} \leq 7.18)^{22,23}$, irrespective of the type of acidosis, was more influential on outcomes than pure severe metabolic acidaemia.

Since severe acidosis was both a negative prognostic factor and a potential indication for SB administration ${ }^{17}$, SB use may thus become an epiphenomenon of severe acidosis and could have introduced the bias of "confounding by indication," leading to worse outcomes associated with SB use $\mathrm{e}^{24-27}$. When the confounding effects of blood $\mathrm{pH}$ were accounted for, the SB use itself was no longer associated with IHCA outcomes, as shown in the Primary Model in the Table 3.

\section{SB use and intra-arrest blood $\mathrm{pH}$}

Furthermore, we attempted to investigate whether the effects of SB would be dependent on intra-arrest blood $\mathrm{pH}$. As shown in Table 3, SB use $\times$ blood $\mathrm{pH} \leq 7.18$ (OR 0.29) and non-SB use $\times$ blood $\mathrm{pH} \leq 7.18$ (OR 0.20 ) were both associated with worse neurological recovery. Nevertheless, the OR of blood $\mathrm{pH} \leq 7.18$ itself was 0.24 , which was dominant in the interaction terms of $\mathrm{SB}$ use $\times$ blood $\mathrm{pH} \leq 7.18$ and non-SB use $\times$ blood $\mathrm{pH} \leq 7.18$ and led to an OR less than one for both interaction terms. Since SB use $\times$ blood $\mathrm{pH} \leq 7.18$ or non-SB use $\times$ blood $\mathrm{pH} \leq 7.18$ was compared with the reference of blood $\mathrm{pH}>7.18$, it would not be possible for SB use to increase the OR of 


\begin{tabular}{|c|c|c|c|}
\hline Independent Variable ${ }^{a}$ & Odds Ratio & 95\% Confidence Interval & $p$ value \\
\hline \multicolumn{4}{|l|}{ Primary Model } \\
\hline $\mathrm{CPR}^{\mathrm{b}}$ duration $(\mathrm{min})$ & 0.91 & $0.88-0.94$ & $<0.001$ \\
\hline Post-ROSC ${ }^{\mathrm{c}}$ percutaneous coronary intervention & 10.47 & $3.97-27.60$ & $<0.001$ \\
\hline Blood $\mathrm{pH} \leqq 7.18$ & 0.24 & $0.11-0.52$ & $<0.001$ \\
\hline Favourable neurological status $24 \mathrm{~h}$ before cardiac arrest & 2.80 & $1.46-5.38$ & 0.002 \\
\hline Age (years) & 0.97 & $0.95-0.99$ & 0.002 \\
\hline Metastatic cancer or any blood-borne malignancy & 0.18 & $0.06-0.55$ & 0.003 \\
\hline Baseline evidence of motor, cognitive, or functional deficits & 0.32 & $0.14-0.73$ & 0.007 \\
\hline Post-ROSC targeted temperature management & 9.46 & $1.66-54.01$ & 0.01 \\
\hline Pneumonia & 0.37 & $0.16-0.87$ & 0.02 \\
\hline Pulmonary artery catheter in place at time of arrest & 21.16 & $1.68-267.36$ & 0.02 \\
\hline $\mathrm{SB}^{\mathrm{d}}$ use & 1.21 & $0.61-2.40$ & 0.58 \\
\hline \multicolumn{4}{|l|}{ Primary Model With Interaction Terms } \\
\hline CPR duration $(\min )$ & 0.90 & $0.87-0.93$ & $<0.001$ \\
\hline Post-ROSC percutaneous coronary intervention & 10.81 & $4.07-28.69$ & $<0.001$ \\
\hline Age between 23 and 69 (years) & 3.40 & $1.73-6.70$ & $<0.001$ \\
\hline Metastatic cancer or any blood-borne malignancy & 0.15 & $0.05-0.48$ & 0.001 \\
\hline Favourable neurological status $24 \mathrm{~h}$ before cardiac arrest & 2.87 & $1.49-5.56$ & 0.002 \\
\hline Non-SB use $\times$ blood $\mathrm{pH} \leqq 7.18$ & 0.20 & $0.06-0.60$ & 0.004 \\
\hline Baseline evidence of motor, cognitive, or functional deficits & 0.30 & $0.13-0.70$ & 0.005 \\
\hline SB use $\times$ blood $\mathrm{pH} \leqq 7.18$ & 0.29 & $0.11-0.76$ & 0.008 \\
\hline Post-ROSC targeted temperature management & 11.74 & $1.68-81.93$ & 0.01 \\
\hline $\mathrm{SB}$ use $\times$ time to $\mathrm{SB} \geqq 20(\mathrm{~min})$ & 6.16 & $1.42-26.75$ & 0.02 \\
\hline Pneumonia & 0.35 & $0.15-0.82$ & 0.02 \\
\hline Pulmonary artery catheter in place at time of arrest & 23.95 & $1.62-353.85$ & 0.02 \\
\hline
\end{tabular}

Table 3. Multiple Logistic Regression Model With Favourable Neurological Outcome at Hospital Discharge as the Dependent Variable. Primary model: goodness-of-fit assessment: $n=1060$, adjusted generalised $R^{2}=0.40$,estimated area under the receiver operating characteristic curve $=0.91$, and Hosmer and Lemeshow goodness-of-fit Chi-Squared test $p<0.001$; Primary model with interaction terms: goodness-of-fit assessment: $\mathrm{n}=1060$, adjusted generalised $R^{2}=0.43$, estimated area under the receiver operating characteristic curve $=0.92$, and Hosmer and Lemeshow goodness-of-fit Chi-Squared test $p<0.001{ }^{a}$ The display of independent variables is arranged in order of $p$ value. ${ }^{b} \mathrm{CPR}$, cardiopulmonary resuscitation. ${ }^{\mathrm{c}} \mathrm{ROSC}$, return of spontaneous circulation. ${ }^{\mathrm{S}} \mathrm{SB}$, sodium bicarbonate.

$\mathrm{SB}$ use $\times$ blood $\mathrm{pH} \leq 7.18$ to greater than one. If $\mathrm{SB}$ use $\times$ blood $\mathrm{pH} \leq 7.18$ was greater than one, this would mean that the neurological outcomes of patients with blood $\mathrm{pH} \leq 7.18$ who received $\mathrm{SB}$ would be better than those with blood $\mathrm{pH}>7.18$, which was not expected. Therefore, in the interpretation of this interaction analysis, we should focus on whether SB use could improve the inherently poor neurological outcomes of patients with blood $\mathrm{pH} \leq 7.18$.

When we examined the two interaction terms, SB use $\times$ blood $\mathrm{pH} \leq 7.18(\mathrm{OR} 0.29)$ and non-SB use $\times$ blood $\mathrm{pH} \leq 7.18$ (OR 0.20 ), we noticed that SB administration actually increased the chances of neurological recovery for patients with blood $\mathrm{pH} \leq 7.18$ (OR 0.24). The increase in ORs between SB use $\times$ blood $\mathrm{pH} \leq 7.18$ and non$\mathrm{SB}$ use $\times$ blood $\mathrm{pH} \leq 7.18$ was caused by the administration of $\mathrm{SB}$, which suggested that for patients with blood $\mathrm{pH} \leq 7.18$, SB might improve neurological outcomes. Nonetheless, the effect estimates of $\mathrm{SB}$ use $\times$ blood $\mathrm{pH} \leq 7.18$ and non-SB use $\times$ blood $\mathrm{pH} \leq 7.18$ were so close that this result could only indicate a tendency of improvement by $\mathrm{SB}$ use rather than a definite conclusion. However, this result may encourage future investigators to examine whether SB use could improve the outcomes of patients with blood $\mathrm{pH} \leq 7.18$. In contrast, for patients with blood $\mathrm{pH}>7.18$ (Table 4), non-SB use was associated with better survival.

Taken together, the most important message of this interaction analysis was that the administration of SB had better to be guided by blood $\mathrm{pH}$, instead of being used empirically. The negative effects of SB use noted in previous studies ${ }^{24-27}$ may just reflect that SB administration failed to improve the dismal outcomes of the patients with severe acidosis, rather than worsen it. In contrast, for patients without severe acidosis, SB should not be administered empirically.

SB use in prolonged CPR. Guidelines for CPR published in the year $2000^{28}$ suggested that SB could be considered as a last-ditch effort after prolonged CPR had been performed with the confirmed interventions, such as defibrillation, chest compression, intubation, ventilation and vasopressor therapy, already in place and ineffective. As revealed in a paediatric IHCA study ${ }^{29}$, the median CPR duration in the SB group (30 min) was significantly higher than that in the non-SB group $(17 \mathrm{~min})$. Andersen et al. ${ }^{11}$ indicated that, if a medication was more likely to be administered when CPR efforts continued longer, this would tend to bias the results toward 


\begin{tabular}{|c|c|c|c|}
\hline Independent Variable ${ }^{a}$ & Odds Ratio & 95\% Confidence Interval & $p$ value \\
\hline \multicolumn{4}{|l|}{ Secondary Model ${ }^{\mathrm{b}}$} \\
\hline CPR duration $(\mathrm{min})$ & 0.94 & $0.92-0.95$ & $<0.001$ \\
\hline Post-ROSC percutaneous coronary intervention & 5.80 & $2.44-13.80$ & $<0.001$ \\
\hline Hypotension & 0.36 & $0.20-0.64$ & $<0.001$ \\
\hline Pulmonary artery catheter in place at time of arrest & 24.56 & $3.12-193.32$ & 0.002 \\
\hline Hepatic insufficiency & 0.38 & $0.18-0.80$ & 0.01 \\
\hline Shockable rhythm & 1.94 & $1.13-3.34$ & 0.02 \\
\hline Metastatic cancer or any blood-borne malignancy & 0.48 & $0.26-0.89$ & 0.02 \\
\hline Blood $\mathrm{pH} \leqq 7.18$ & 0.61 & $0.38-0.98$ & 0.04 \\
\hline SB use & 0.70 & $0.44-1.12$ & 0.14 \\
\hline \multicolumn{4}{|l|}{ Secondary Model With Interaction Terms ${ }^{\mathrm{c}}$} \\
\hline CPR duration (min) & 0.94 & $0.92-0.95$ & $<0.001$ \\
\hline Post-ROSC percutaneous coronary intervention & 5.16 & $2.15-12.39$ & $<0.001$ \\
\hline Hypotension & 0.37 & $0.21-0.67$ & 0.001 \\
\hline Metastatic cancer or any blood-borne malignancy & 0.44 & $0.24-0.81$ & 0.003 \\
\hline Shockable rhythm & 1.98 & $1.15-3.40$ & 0.005 \\
\hline Pulmonary artery catheter in place at time of arrest & 26.50 & $3.34-210.17$ & 0.009 \\
\hline Hepatic insufficiency & 0.40 & $0.19-0.83$ & 0.009 \\
\hline Non-SB use $\times$ blood $\mathrm{pH}>7.18$ & 1.82 & $1.12-2.95$ & 0.02 \\
\hline Favourable neurological status $24 \mathrm{~h}$ before cardiac arrest & 1.56 & $1.01-2.41$ & 0.05 \\
\hline
\end{tabular}

Table 4. Multiple Logistic Regression Model With Survival at Hospital Discharge as the Dependent Variable. Abbreviations: CPR, cardiopulmonary resuscitation; ROSC, return of spontaneous circulation; SB, sodium

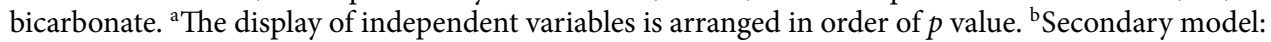
goodness-of-fit assessment: $\mathrm{n}=1060$, adjusted generalised $R^{2}=0.34$,estimated area under the receiver operating characteristic curve $=0.85$, and Hosmer and Lemeshow goodness-of-fit Chi-Squared test $p=0.10$. ${ }^{\mathrm{c} S e c o n d a r y}$ model with interaction terms: goodness-of-fit assessment: $\mathrm{n}=1060$, adjusted generalised $R^{2}=0.36$, estimated area under the receiver operating characteristic curve $=0.85$, and Hosmer and Lemeshow goodness-of-fit ChiSquared test $p=0.18$.

a harmful effect since CPR duration was strongly associated with worse outcomes ${ }^{30,31}$. This bias might be mitigated to some extent by considering both CPR duration and timing of intervention in the analysis. As shown in Table 3, both CPR duration and time to SB were analysed, revealing that when CPR persisted longer than $20 \mathrm{~min}, \mathrm{SB}$ use may be beneficial.

The definition of "prolonged CPR" was not clearly defined in the 2000 guidelines for CPR ${ }^{28}$. Vukmir et al. ${ }^{26}$ demonstrated that overall, there was no difference in survival between the SB and placebo groups. Nonetheless, survival rates were significantly higher in the SB group than in the placebo group in the subgroup with prolonged arrest $(>15 \mathrm{~min})^{23,26}$. According to the 3 -phase cardiac arrest model ${ }^{32}$, CPR duration longer than 20 min may fall into the metabolic phase, during which interventions other than prompt defibrillation or high-quality CPR should be employed, such as SB. Beyond its metabolic effects, SB was also a volume expander, which might be beneficial during circulatory phase. Nonetheless, since we did not collect the hemodynamic data during CPR, the circulatory effects of SB during CPR should be further examined. Furthermore, despite the fact that our results showed that SB use in prolonged CPR might be effective, it should be remembered that SB is administered after other effective interventions have been performed ${ }^{28}$.

In summary, SB tended to be used in a subgroup with inherently poor outcomes. By carefully dealing with the bias of "confounding by indication" and "resuscitation time bias," our results seemed to corroborate SB use in certain conditions. However, because of the retrospective study design, the results should be viewed as hypothesis-generating, laying the foundation for further clinical trials.

Study limitations. First, because of the inherent limitation of the retrospective study design, only an association, rather than a causal relationship, could be investigated between independent and outcome variables. The effects of unmeasured confounders could have introduced bias into the results. Second, it was difficult to ascertain whether blood gas was retrieved from an arterial or a venous origin. It was reported that venous $\mathrm{pH}$ may be consistent with arterial $\mathrm{pH}^{33}$. However, this could only be resolved by a prospective study adopting a certified method to obtain arterial blood gas. Third, the SB dosage was not accounted for in the analysis. A doseresponse effect, if present, could have offered stronger evidence for the effect of SB use on outcomes. Fourth, the exact timing of obtaining blood gas samples was not recorded in the previously established IHCA database ${ }^{12,13}$ and therefore, we could not verify whether the timing of obtaining blood gas samples was prior to the timing of SB use. Nonetheless, since the clinicians in NTUH were instructed to obtain blood gas analysis as soon as possible in the beginning of CPR, the interval between the timing of blood gas analysis and SB use may be quite small (maximum: $10 \mathrm{~min}$ ), which may not cause significant changes in blood gas analysis data. Finally, we 


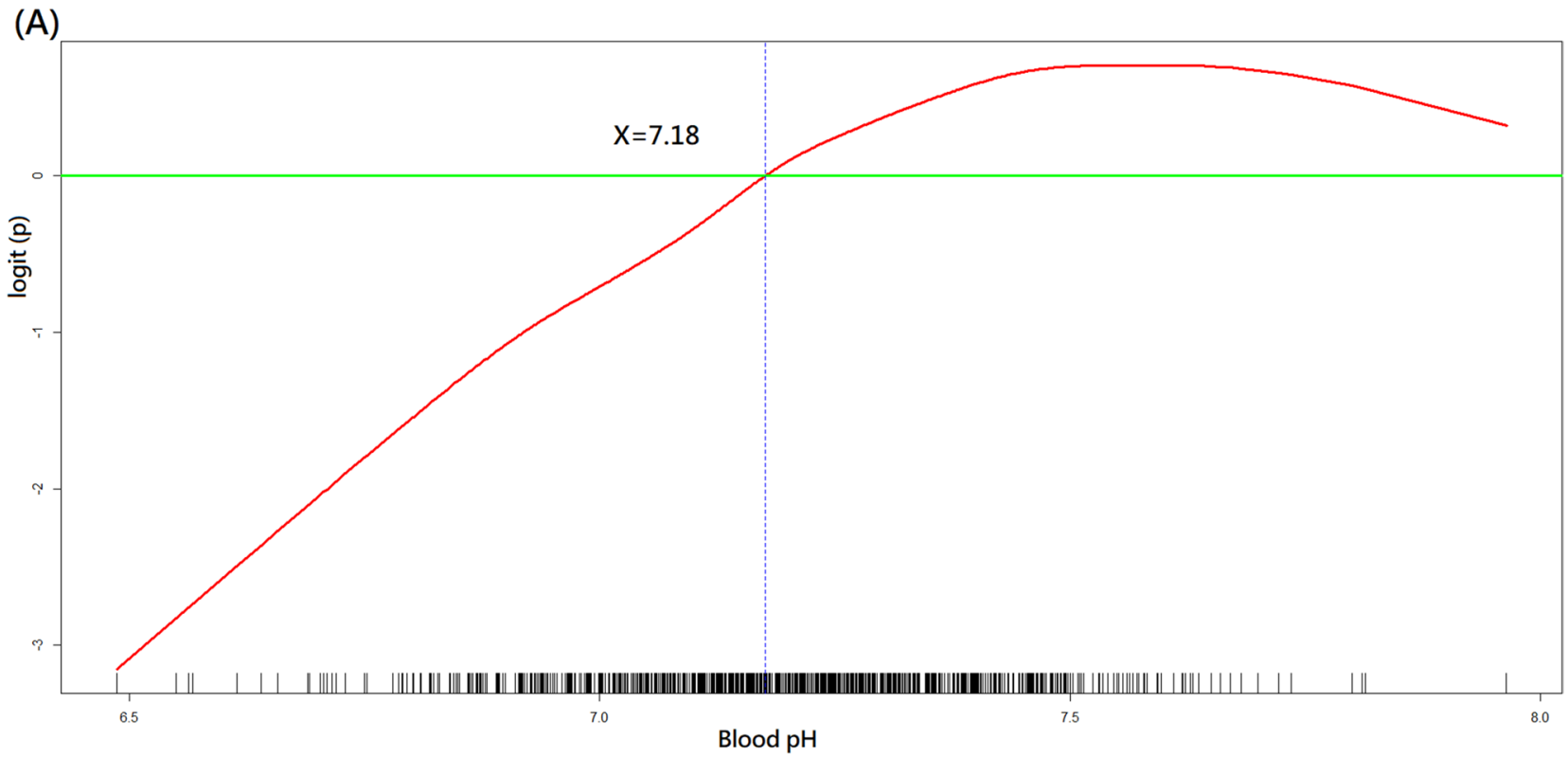

(B)

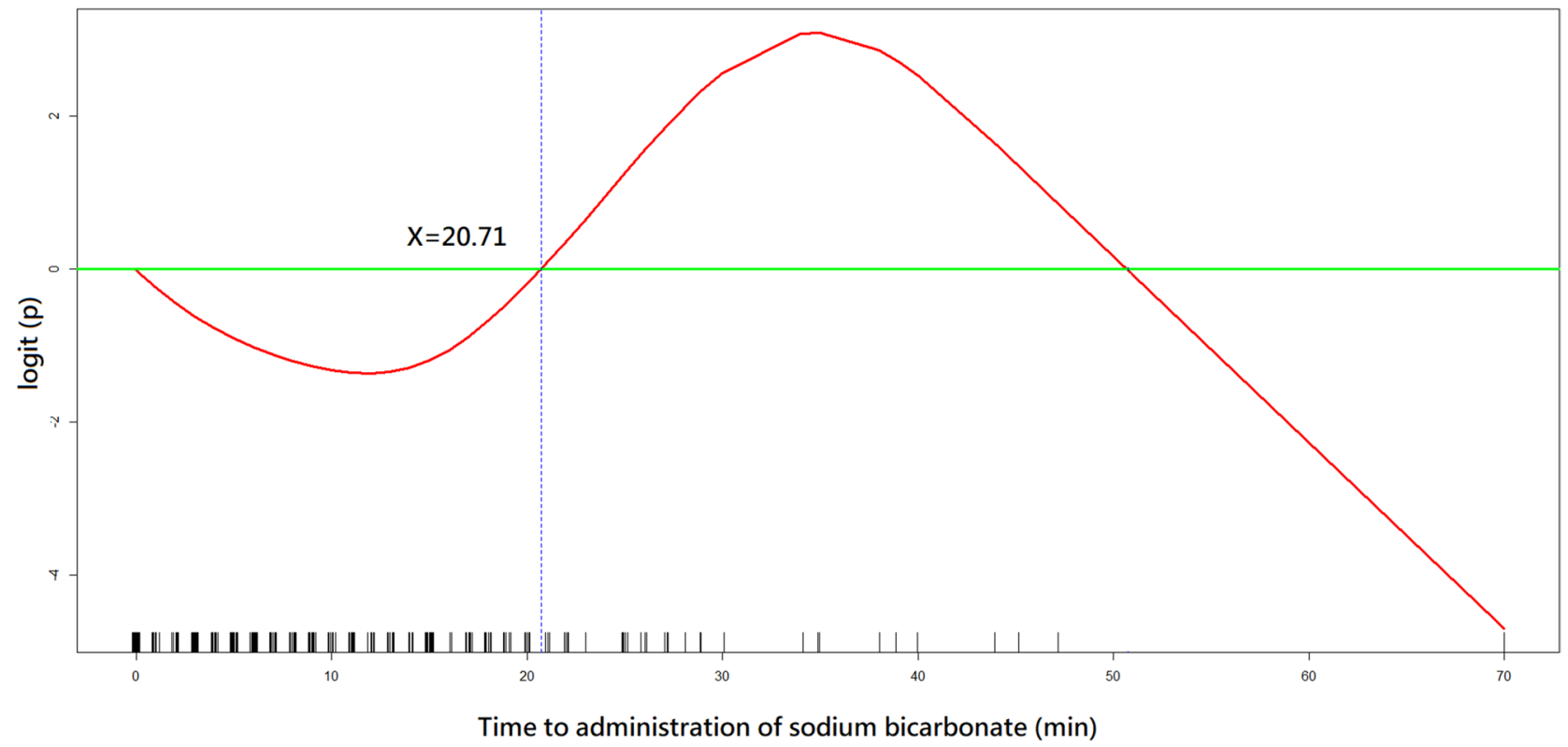

Figure 1. GAM plots. Nonparametric modelling of the effect of blood $\mathrm{pH}$ and time to administration of sodium bicarbonate on the logit of probability for favourable neurological outcome at hospital discharge. GAM, generalised additive model.

studied only patients with intra-arrest blood gas data available. As shown in Supplemental Table 1 and 2, there were some differences between patients included and not included in the analysis, leading to worse survival and neurological outcomes in patients in current analysis. Therefore, our results may only be applied to patients for whom clinicians would order blood gas analysis. Our results may not be applied to patients for whom clinicians would not order blood gas analysis and also did not suggest that all IHCA patients should receive blood gas analysis. Whether all IHCA patients would benefit from early intra-arrest blood gas analysis should be further examined in prospective studies. Therefore, our results could only be applied to patients who received blood gas analysis during CPR and these results need further examination before they could be generalized to all patients undergoing CPR.

\section{Conclusions}

Our results suggested that SB should not be empirically administered for IHCA patients with blood pH>7.18. Also, for patients receiving CPR longer than $20 \mathrm{~min}$, SB might be administered, given other effective interventions had been performed. 


\section{Data availability}

The data that support the findings of this study are available on request from the corresponding author, WenJone Chen.

Received: 10 December 2020; Accepted: 17 May 2021

Published online: 11 June 2021

\section{References}

1. Benjamin, E. J. et al. Heart disease and stroke statistics-2017 update: A report from the American Heart Association. Circulation 135, e146-e603 (2017).

2. Lin, C. C. et al. Association between acidosis and outcome in out-of-hospital cardiac arrest patients. Am. J. Emerg. Med. 36, 2309-2310 (2018).

3. Shin, J. et al. Initial blood $\mathrm{pH}$ during cardiopulmonary resuscitation in out-of-hospital cardiac arrest patients: A multicenter observational registry-based study. Crit. Care 21, 322 (2017).

4. Wang, C. H. et al. Associations between early intra-arrest blood acidaemia and outcomes of adult in-hospital cardiac arrest: A retrospective cohort study. J. Formos. Med. Assoc. 119, 644-651 (2020).

5. Standards for Cardiopulmonary Resuscitation (CPR) and Emergency Cardiac Care (ECC). JAMA 227, 833-68 (1974).

6. Soar, J. et al. European resuscitation council guidelines for resuscitation 2015: Section 3. Adult advanced life support. Resuscitation 95, 100-147 (2015).

7. Link, M. S. et al. Part 7: Adult Advanced Cardiovascular Life Support: 2015 American Heart Association Guidelines Update for Cardiopulmonary Resuscitation and Emergency Cardiovascular Care. Circulation 132, S444-S464 (2015).

8. Moskowitz, A. et al. Trends over time in drug administration during adult in-hospital cardiac arrest. Crit. Care Med. 47, 194-200 (2019).

9. Geraci, M. J. et al. Prevalence of sodium bicarbonate-induced alkalemia in cardiopulmonary arrest patients. Ann. Pharmacother 43, 1245-1250 (2009).

10. Bar-Joseph, G. et al. Clinical use of sodium bicarbonate during cardiopulmonary resuscitation-is it used sensibly?. Resuscitation 54, 47-55 (2002).

11. Andersen, L. W., Grossestreuer, A. V. \& Donnino, M. W. "Resuscitation time bias"-A unique challenge for observational cardiac arrest research. Resuscitation 125, 79-82 (2018).

12. Wang, C. H. et al. Associations between body size and outcomes of adult in-hospital cardiac arrest: A retrospective cohort study. Resuscitation 130, 67-72 (2018).

13. Wang, C. H. et al. The association between long-term glycaemic control, glycaemic gap and neurological outcome of in-hospital cardiac arrest in diabetics: A retrospective cohort study. Resuscitation 133, 18-24 (2018).

14. ECC Committee, Subcommittees and Task Forces of the American Heart Association. 2005 American Heart Association Guidelines for Cardiopulmonary Resuscitation and Emergency Cardiovascular Care. Circulation 112, IV1-203 (2005).

15. Field, J. M. et al. Part 1: Executive summary: 2010 American Heart Association Guidelines for Cardiopulmonary Resuscitation and Emergency Cardiovascular Care. Circulation 122, S640-S656 (2010).

16. Jacobs, I. et al. Cardiac arrest and cardiopulmonary resuscitation outcome reports: Update and simplification of the Utstein templates for resuscitation registries: A statement for healthcare professionals from a task force of the International Liaison Committee on Resuscitation (American Heart Association, European Resuscitation Council, Australian Resuscitation Council, New Zealand Resuscitation Council, Heart and Stroke Foundation of Canada, InterAmerican Heart Foundation, Resuscitation Councils of Southern Africa). Circulation 110, 3385-3397 (2004).

17. Jaber, S. et al. Sodium bicarbonate therapy for patients with severe metabolic acidaemia in the intensive care unit (BICAR-ICU): A multicentre, open-label, randomised controlled, phase 3 trial. Lancet 392, 31-40 (2018).

18. Becker, L. B. et al. Primary outcomes for resuscitation science studies: A consensus statement from the American Heart Association. Circulation 124, 2158-2177 (2011).

19. Hastie, T. J. \& Tibshirani, R. J. Generalized Additive Models (Chapman \& Hall, London, 1990).

20. Velissaris, D. et al. Use of sodium bicarbonate in cardiac arrest: Current guidelines and literature review. J. Clin. Med. Res. 8, 277-283 (2016).

21. Wang, C. H. et al. The effects of calcium and sodium bicarbonate on severe hyperkalaemia during cardiopulmonary resuscitation: A retrospective cohort study of adult in-hospital cardiac arrest. Resuscitation 98, 105-111 (2016).

22. Kraut, J. A. \& Kurtz, I. Use of base in the treatment of acute severe organic acidosis by nephrologists and critical care physicians: Results of an online survey. Clin. Exp. Nephrol. 10, 111-117 (2006).

23. Kraut, J. A. \& Madias, N. E. Metabolic acidosis: Pathophysiology, diagnosis and management. Nat. Rev. Nephrol. 6, 274-285 (2010).

24. van Walraven, C. et al. Do advanced cardiac life support drugs increase resuscitation rates from in-hospital cardiac arrest? The OTAC Study Group. Am. J. Emerg. Med. 32, 544-553 (1998).

25. Aufderheide, T. P. et al. Prehospital bicarbonate use in cardiac arrest: A 3-year experience. Am. J. Emerg. Med. 10, 4-7 (1992).

26. Vukmir, R. B., Katz, L. \& Sodium Bicarbonate Study Group. Sodium bicarbonate improves outcome in prolonged prehospital cardiac arrest. Am. J. Emerg. Med. 24, 156-61 (2006).

27. Kawano, T. et al. Prehospital sodium bicarbonate use could worsen long term survival with favorable neurological recovery among patients with out-of-hospital cardiac arrest. Resuscitation 119, 63-69 (2017).

28. Part 6: Advanced Cardiovascular Life Support. Circulation 102, I-129-I-135 (2000).

29. Raymond, T. T. et al. Sodium bicarbonate use during in-hospital pediatric pulseless cardiac arrest-A report from the American Heart Association Get With The Guidelines-Resuscitation. Resuscitation 89, 106-113 (2015).

30. Chan, P. S. et al. A validated prediction tool for initial survivors of in-hospital cardiac arrest. Arch. Intern. Med. 172, 947-953 (2012).

31. Wang, C. H. et al. Validation of the Cardiac Arrest Survival Postresuscitation In-hospital (CASPRI) score in an East Asian population. PLoS One 13, e0202938 (2018).

32. Weisfeldt, M. L. \& Becker, L. B. Resuscitation after cardiac arrest: A 3-phase time-sensitive model. JAMA 288, 3035-3038 (2002).

33. Rang, L. C. et al. Can peripheral venous blood gases replace arterial blood gases in emergency department patients?. CJEM 4, 7-15 (2002).

\section{Acknowledgements}

We thank Centre of Quality Management of National Taiwan University Hospital for providing the list of patients sustaining in-hospital cardiac arrest. We thank the staff of the $3^{\text {rd }}$ Core Lab, Department of Medical Research, National Taiwan University Hospital for technical support. Author Chih-Hung Wang recieved a grant (110S4808) from the National Taiwan University Hospital. Author Wen-Jone Chen received a grant (110-S4935) from the National Taiwan University Hospital. National Taiwan University Hospital had no involvement in designing 
the study, collecting, analysing or interpreting the data, writing the manuscript, or deciding whether to submit the manuscript for publication.

\section{Author contributions}

C.H.W., W.T.C., and C.H.H. conceived the idea and drafted the manuscript. C.Y.W. and MCW performed the analysis. M.S.T., T.C.L., E.C., and Y.L.H. interpreted the results and helped to revise the manuscript. W.J.C. helped to frame the idea of the study and helped to analyse the data. All authors read and approved the final manuscript.

\section{Competing interests}

The authors declare no competing interests.

\section{Additional information}

Supplementary Information The online version contains supplementary material available at https://doi.org/ 10.1038/s41598-021-91936-3.

Correspondence and requests for materials should be addressed to W.-J.C.

Reprints and permissions information is available at www.nature.com/reprints.

Publisher's note Springer Nature remains neutral with regard to jurisdictional claims in published maps and institutional affiliations.

(c) (1) Open Access This article is licensed under a Creative Commons Attribution 4.0 International License, which permits use, sharing, adaptation, distribution and reproduction in any medium or format, as long as you give appropriate credit to the original author(s) and the source, provide a link to the Creative Commons licence, and indicate if changes were made. The images or other third party material in this article are included in the article's Creative Commons licence, unless indicated otherwise in a credit line to the material. If material is not included in the article's Creative Commons licence and your intended use is not permitted by statutory regulation or exceeds the permitted use, you will need to obtain permission directly from the copyright holder. To view a copy of this licence, visit http://creativecommons.org/licenses/by/4.0/.

(C) The Author(s) 2021 